\title{
La cryptorchidie chez l'enfant sénégalais en âge scolaire
}

\author{
S.M. Gueye, M. BA, A. Ndoye, C. Sylla, P.A. Falı, A. Mensah
}

Clinique Urologique (Pr A. Mensah), CHU Aristide Le Dantec, BP 6039, Dakar, Sénégal

\section{RESUME}

Le but de cette enquête épidémiologique prospective a été de déterminer la prévalence et de préciser les particularités de la cryptorchidie chez le garçon sénégalais en âge scolaire.

Sur 2830 garçons examinés, nous avons découvert 40 cas de testicule non descendu dont 19 à gauche, 17 à droite et 4 bilatéraux. La prévalence est de $1,41 \%$. Par ailleurs, nous avons trouvé une corrélation entre la présence d'un testicule non descendu à l'âge scolaire et la catégorie socioprofessionnelle des parents. En effet, elle varie de $0,25 \%$ chez les enfants issus de milieu socialement favorisés à $2,5 \%$ chez les enfants défavorisés en particulier ceux d'ouvriers. Nous pouvons conclure que la prévalence de la cryptorchidie à l'âge scolaire est plus élevée dans notre étude que celles rapportées dans la littérature $[10,28]$. Le défaut d'information des parents issus de milieu socio-économique défavorisé, et la difficulté d'accès aux structures médicalisées semblent être les principaux facteurs de retard de dépistage et partant de traitement des testicules non descendus.

Mots clés : Cryptorchidie, Testicule non descendu, Infertilité.

\section{INTRODUCTION}

S'il est bien établi que le testicule ne peut assurer correctement ses fonctions exocrine et endocrine que lorsque sa descente est complète dans le scrotum [2], les mécanismes intimes qui régulent cette migration testiculaire, ainsi que l'étio-pathogénie de la cryptorchidie sont encore du domaine des hypothèses. Le rôle probable joué d'une part par le gubernaculum testis, le nerf génito-fémoral et la Calcitonin Gene-Related Peptide (CG-RP), d'autre part par les anomalies épididymaires associées a ouvert de nouveaux axes de recherche tendant à expliquer l'arrêt de migration du testicule $[4,12,25,32]$. La relation entre l'infertilité masculine et la cryptorchidie inspire encore d'importantes recherches expérimentales et cliniques $[3,7,16,24,27]$. L'effet néfaste de l'hyperthermie et son implication dans la pathogénie de l'infertilité associée à la cryptorchidie offrent des perspectives intéressantes dans le domaine de la contraception masculine [24]. En outre, les particularités des tumeurs malignes sur testicule non descendus ne sont pas bien définies, même si le risque relatif autrefois estimé entre 30 et 40 , est actuellement revu à la baisse et ne dépasserait pas 10 [5, 9, 13, 18, 22, 26].

Afin de minorer le risque de survenue de ces complications, l'abaissement du testicule doit se faire avant la fin de la deuxième année de vie $[7-9,11,13,14,18,19,23]$. Malheureusement, les enfants porteurs de 
dystopies testiculaires nous arrivent encore très tard à un âge dépassant de loin ce délai théorique de deux ans. Ainsi, avons nous entrepris ce travail dont les objectifs sont d'une part d'étudier la prévalence des cryptorchidies chez le garçon sénégalais en âge scolaire, d'autre part de sensibiliser les parents et les praticiens sur la nécessité d'un dépistage précoce de l'anomalie.

\section{MATERIEL ET METHODES}

Il s'agit d'une étude prospective réalisée sous forme d'une enquête sur le terrain. L'enquête a eu lieu dans cinq écoles élémentaires de la Commune de Thiès : E1= I. Sarr, E2= A2. Lo, E3= D. Brottier, E4= M.Thiam, E5= I.Diop.

Les 5 écoles ont été choisies dans cinq zones de niveaux socio-économiques différents afin de toucher toutes les couches de la population de la commune.

Une lettre d'intention a été adressée à chaque directeur d'école qui s'est chargé d'informer les maîtres, les élèves et leurs parents des objectifs et des modalités de notre enquête.

Pour chaque enfant a été remplie une fiche de renseignements où figuraient les paramètres suivants : l'âge de l'enfant, la taille de la fratrie (le nombre de frères et sœurs), la catégorie socioprofessionnelle des parents, en particulier celle du père. Chez les garçons présentant une cryptorchidie, le côté de l'anomalie, la position et le volume du testicule non descendu, s'il est palpable, le volume du testicule controlatéral, et les anomalies associées ont été notés.

2830 élèves ont été examinés, parmi lesquels 40 ont été trouvés porteurs d'une cryptorchidie.

L'analyse statistique des données recueillies a été faite selon la méthode des cas témoins.

\section{RESULTATS}

Quarante enfants ont été trouvés porteurs d'un testicule non descendu. L'histogramme I représente la répartition des enfants porteurs d'un testicule non descendu suivant les différentes écoles. La dispersion des âges se faisait entre 6 et 17 ans. 5\% des enfants porteurs de cryptorchidie avaient un retard scolaire (Figure 1). L'anomalie siégeait 19 fois à gauche, 17 fois à droite, et était bilatérale dans 4 cas. Le testicule non descendu était palpé 15 fois en position inguinale, une fois en position pré-scrotale. Le testicule n'était pas palpable dans 28 cas.

Certaines anomalies associées au testicule non descendu ont été notées (Figure 2). Il s'agissait d'une persistance d'un canal péritonéovaginal qui se manifestait soit par une hydrocèle $(40 \%)$, soit par une hernie $(26,67 \%)$.

Parfois nous avons trouvé une malformation congénitale de la verge telle un hypospadias $(6,67 \%)$ ou une torsion de la verge $(6,67 \%)$. Dans d'autres cas il s'agissait d'une anomalie acquise suite à un accident de la circoncision comme une sténose du méat urétral $(13,33 \%)$ ou une amputation du gland $(6,67 \%)$.

La prévalence brute des testicules non descendus dans notre échantillon était de $1,41 \%$. Cette prévalence variait selon le niveau socio-économique de l'enfant. Elle était élevée chez les enfants d'ouvriers $(2,5 \%)$, moyenne chez les enfants d'artisans, de commerçants ou d'agents de l'administration $(1,2 \%)$, et faible chez les enfants de cadres $(0,25 \%)$.

Les enfants d'ouvriers représentaient $45 \%$ de ceux présentant l'anomalie, et $29 \%$ des enfants non porteurs de l'anomalie. La proportion des enfants de cadres parmi ceux porteurs d'un testicule non descendu était de $3 \%$. Cette proportion était de $12 \%$ dans le groupe des enfants témoins. 


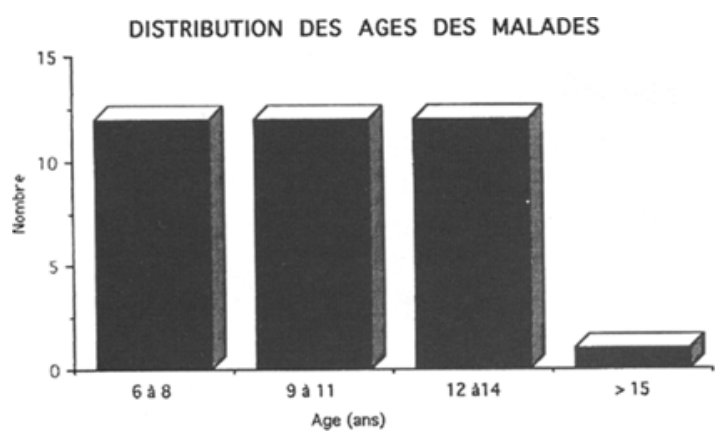

Figure 1 : Distribution des âges des malades.

\section{COMMENTAIRES}

La cryptorchidie est l'une des plus fréquentes anomalies congénitales de l'appareil uro-génital. Sa prévalence théorique varie selon l'âge. Estimée entre 9 et $30 \%$ chez le nouveau-né prématuré, elle se situe entre 3,4 à $5,8 \%$ chez le nouveau-né à terme, pour se stabiliser à $0,8 \%$ à la fin de la première année de vie. La prévalence de $1,41 \%$ que nous avons retrouvée dans notre échantillon est au dessus des chiffres rapportés dans les rares études portant sur l'épidémiologie des testicules non descendus à l'âge scolaire. En effet, Scorer et Farrington [28] examinant 3612 nouveau-nés ont trouvé une prévalence différente selon que l'enfant soit né à terme ou prématurément. Chez les 1500 nouveau-nés à terme, $3,4 \%$ d'entre eux avaient un testicule cryptorchide, tandis que chez les 142 prématurés $30,3 \%$ étaient porteurs de l'anomalie. Ils ont aussi trouvé que plus le poids de naissance était faible, plus l'incidence de la cryptorchidie était élevée. En effet, l'anomalie était trouvée chez $68,5 \%$ des prématurés de moins de 1800 grammes. Ce taux était de $100 \%$ quand le poids de naissance était inférieur à 900 grammes. Cependant, lorsque ces prématurés commencent à prendre du poids après la naissance, cer-
REPARTITION DES ANOMALIES ASSOCIEES

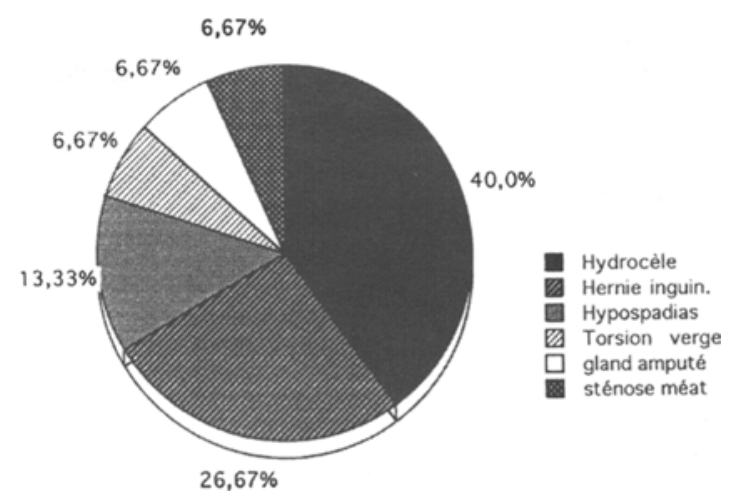

Figure 2 : Répartition des anomalies associées.

tains testicules peuvent compléter leur descente spontanée avant la fin de la première année de vie, âge à laquelle la prévalence se stabilise à $0,8 \%$. Ce taux ne varie plus et reste identique à la puberté.

Dans une étude réalisée chez des enfants d'âges superposables à notre série, CourPalais [10] rapporte une prévalence de la cryptorchidie vraie de $0,75 \%$ à 5 ans, $0,95 \%$ à 8 ans, et $0,64 \%$ à 11 ans. Ces taux sont en deçà de celui que nous avons trouvé.

Nous avons essayé de voir si une corrélation existait entre le niveau socio-économique et la présence d'un testicule non descendu à l'âge scolaire. La prédominance des enfants d'ouvriers parmi ceux présentant l'anomalie est évidente. De plus, bien que nous retrouvons quelques cas de cryptorchidie chez les enfants issus d'un milieu social favorisé, la prévalence dans ce groupe est faible à l'âge scolaire. Ainsi, il apparaît que si un faible niveau socio-économique n'expose pas au risque de survenue d'une cryptorchidie, il constitue un facteur important de retard diagnostique. Ce retard de dépistage de l'anomalie a été rapporté par Tshitala et al [29] qui, dans une série de 131 patients porteurs d'un testicule non descendu, on trouvé que $64,45 \%$ avaient plus de 6 ans. 
Tableau 1 : Répartition des cas selon les écoles.

\begin{tabular}{lcc}
\hline Ecoles & Effectifs & Nbre de cas \\
\hline E1 & 714 & 2 \\
E2 & 609 & $\mathbf{1 6}$ \\
E3 & 497 & 6 \\
E4 & 462 & 6 \\
E5 & 548 & 10 \\
Total & $\mathbf{2 8 3 0}$ & $\mathbf{4 0}$ \\
\hline
\end{tabular}

Cela est probablement dû au manque d'informations des parents et leur difficulté d'accéder aux structures médicalisées. A l'opposé, un niveau socio-économique élevé facilite l'accès à l'information médicale et aux structures de soins, d'où la plus grande probabilité de découverte précoce de l'anomalie.

Dans notre série, l'âge n'est pas un facteur discriminant car l'échantillon a été choisi dans une population d'élèves scolarisés au cycle primaire. Nous avons noté une légère prédominance non significative de la localisation à gauche de l'anomalie, alors que la prédominance droite est habituellement signalée dans la littérature $[6,19,29,31]$.

Quant à la position des testicules non descendus à l'examen clinique, elle ne reflétait pas forcément la position réelle du testicule telle que découverte lors de l'intervention. Les conditions de l'examen rendaient parfois difficile l'obtention d'une bonne relaxation musculaire, ce qui pourrait expliquer, du moins en partie, la forte proportion de testicules non palpables.

Des anomalies associées aux cryptorchidies sont souvent rapportées dans la littérature $[1,15,20,30]$. Elles peuvent être mineures comme une persistance d'un canal péritonéo-vaginal, ou majeures à type de différenciation masculine incomplète des organes génitaux externes pouvant faire évoquer, lorsque la dystopie testiculaire est bilatérale, un pseudo-hermaphrodisme masculin voir un hermaphrodisme vrai.
Krueger et al [20] rapportent une prévalence de $12 \%$ chez des garçons porteurs de valves de l'urètre postérieur. Ailleurs, l'association d'une cryptorchidie à des anomalies de la paroi abdominale est souvent décrite. Aliotta et al [1] ont trouvé une cryptorchidie chez $7,7 \%$ des patients présentant une omphalocèle et $25 \%$ chez ceux nés avec une laparoschisis. Le risque relatif pour ces deux affections est estimé respectivement à 1,34 et 4,37 .

De cette étude il ressort qu'un niveau socio-économique faible est un facteur bien corrélé au retard de diagnostic des cryptorchidies. C'est dire tous les efforts qui restent à faire dans le dépistage des anomalies uro-génitales congénitales en général, et des testicules non descendus en particulier. Cette politique de dépistage doit reposer sur une information bien menée en direction des praticiens et des populations. Mieux, la palpation des testicules des nouveau-nés doit être systématique afin de découvrir l'anomalie dès la naissance. Les enfants porteurs d'une cryptorchidie doivent être régulièrement suivis au cours de la première année de vie afin de donner quelque chance à une possible descente spontanée du testicule. Ceux chez lesquels la descente ne se sera pas complétée à l'âge d'un an doivent être opérés dans la deuxième année avant que ne s'installent des lésions histologiques irréversibles qui vont compromettre la fertilité. Cette attitude est la seule à même de minorer les risques de survenue de complications graves que sont l'infertilité et la dégénérescence maligne.

\section{REFERENCES}

1. Aliotrita P.J., Piengonte M., Karp M., Greenfielid S.P. : Cryptorchidism in newborns with Gastroschisis and Omphalocele. Urology, 1992, 40, 1, 8486.

2. ANoukassis C., Liakakos D., Kiburis J., Doulas N.I., PAPASPYROC P. : Effects or surgical repair of cryptorchidism on endoctrine testicular functiun. J. Ped., 1983, 103, 6, 919-921. 
3. ARvis G., NiCOLRT Y. : Cryptorchidie et infertilité. Ann. Urol., 1984, 18, 4, 246-252.

4. BACKHOUSE K.M. : Development and descent of the testis. Eur. J. Ped. 1982, 139, 249-252.

5. Batata M.A. ET AL. : Cryptorchidism and testicular cancer. J. Urol., 1980, 124, 382-388.

6. Beltran-Brown F., Villegas-Ajvarez F. : Clinical classification of undescended testes : Experience in 1010 orchiopexies. J. Ped. Surg., 1988, 23, 5, 444447.

7. CARRIZA C. FT AL. : Testicular maldescent and infertility. Andrologia, 1990, 22, 285-288.

8. Cexidron M. ET Al. : Cryptorchidism, orchiopexy and infertility : A critical long terme retrospective analysis. J. Urol., 1989, 142, 559-562.

9. Chilvers C., Dudley N.E., Gough M.K., JaACKSON M.B., PIKE M.C. : Undescended testes : Effects of treatment on subsequent risk of fertility and malignancy. J. Ped. Surg., 1988, 21, 8, 691-696.

10. Cour-PaI.aIS I.J. : Spontaneous descent of the testicle. Lancet, 1966, 1, 1403.

11.FALLoN B. AND KENNEDY T.J. : Long term follow up fertility in cryptorchid patients. Urology, 1985, 25, $5,502-504$.

12. Gilu B., Kogav S., Stark S., Reda E., LevitT S. : Significance of epididymal and ductal anomalies associated with maldescended testes. J. Urol., 1989, 142, 556-558.

13. Giwercman A., Grinsted J., Hansen B., Jensex O.M., SKakkeback N.E. : Testicular cancer risk in boys with undescended testes : A cohort study. J. Urol., 1987, 138, 1214-1216.

14. Gueye S.M., BA M., Syl.1.A C., DÈye M.L., Diagne B.A., MeNSAH A. : L'orchidopexie in-dartos dans le traitement de la cryptorchidie. J. Chir., 1992, 129, $5,263-265$.

15. Had7.sei.Imovic F. ET AL : Omphalocele, cryptorchidism, and brain malformations. J. Pediatr. Surg., $1987,22,854$.

16. Hakgreave T.B., Elton R.A., Webb J.A., Busuttil, A., Chisholm G.D. : Maldescended testis and fertility : A review of 68 cases. B.J. Urol., 1984, 56, 734-739.

17. HufF D.S., Hadzisflimovic F., SNyder H., DUcKFTT J.W., KeATiNG M.A. : Post-natal testicular maldevelopment in unilateral cryptorchidism. J. Urol., 1989, 142, Part 2, 546-548.

18. JONES B.J. ET AL : Influence of prior orchiopexy on stage and prognosis of testicular cancer. Fur. Urol., 1991, 19, 201-203.
19. KOGAN S.J. : Fertility in cryptorchidism : an overview in 1987. Eur. J. Ped. 1987, 146, (Suppl. 2), S21-S24.

20. Kogan S.J., Tennenbaum S., Gill B., Reim E., LEVITT S. : Efficacy of orchiopexy by patient aged one year for cryptorchidisme. J. Urol., 1990, 144, 508-509.

21. KRLEger R.P., HaRdy B.E., ChLRChILl B.M. : Cryptorchidism in boys with posterior urethral valves. J. Urol., 1980, 124, 101-102.

22. KLLKaRni J.N. ANd Kamat M.R. : Tumors in undescended testis. J. Surg. Oncol., 1991, 46, 257-260.

23. Ludwig G. and Potempa J. : Der optimale zeit punkt der behandlung des kryptorchismus. Deutch Med. Wschr., 1975, 100, 680-683.

24. Mieusset R., Bljan L., Maisat A., Pontonnier F. : Hyperthermie scrotale et infertilité masculine. Progrès en Urol., 1992, 2, 31-36.

25. MLininerg D.T. : The epididymis and testicular descent. Eur. J. Ped., 1987, 146 (Suppl. 2), S28S30.

26. Pike M.C., Chilvers C., Peckham M.J. : Effects of age of orchiopexy on the risk of testicular cancer. Lancet, 1986, 31, 1246-1248.

27. Puri P. and O'Donneli. B. : Semen analysis in patients operated on for impalpable testis. B.J. Urol., 1990, 66, 646-647.

28. Scorer G.C. AND Farrivgton G.H. : Congenital deformities of the testis and epididymis. New York, Appleton-Century-Crofs, 1972, Chap 2.

29. Tshitala B., Tshipeta N., Mptute Y., Llfema L.N. : Notre expérience dans le traitement des cryptorchidies aux cliniques universitaires de Kinshasa. Analyse de 131 cas. Méd. Afr. Noire, 1993, $40,2,108-111$.

30. VIsSkR H.K.A. : Associated anomalies in undescended testes : Eur. J. Pediatr., 1982, 139, 272-274.

31. WhITAKeR R.H. : Undescended testis : The need for a standard classification. B.J. Urol., 1972, 70, 1-6.

32. Wil.itams M.P.L. ANis Huston J.M. : The history and ideas about testicular descent. Ped. Surg. Int. $1991,6,180-184$.

\section{ABSTRACT}

Cryptorchidism in senegalese schoolboys

$$
\text { S.M. Gueye, M. BA, A. Ndoye, C. Sylla, }
$$$$
\text { P. Fall, A. Mensah }
$$ 
The aim of this prospective study was to determine the incidence and the particularities of cryptorchidism in senegalese schoolboys. In a serie of 2830 boys, 40 of them had a cryptorchid testes. The incidence was $1.41 \%$. This rate is higher than those previously reported in the litterature.

Correlating the incidence to the economic level of the boys, it appears that the incidence was very low in rich group $(0.25 \%)$. In the opposite it was high in poor groups $(2.5 \%)$.

We conclude that the high incidence rate of cryptorchidism in boys is due to the lack of information of parents issued from poor social groups.

Key words : Cryptorchidism. Maldescended testes. Infertility. 